\title{
Regulation of Cytoskeletal Functions in Pig Oocytes
}

\author{
Heide Schatten ${ }^{*}$, Randall S. Prather ${ }^{* *}$, Heng-Yu Fan ${ }^{* * *}$, Chao Tong ${ }^{* * *}$, and Qing-Yuan Sun ${ }^{* * *}$ \\ *Dept. of Veterinary Pathobiology, University of Missouri-Columbia, Columbia, MO 65211 \\ ** Dept. of Animal Science, University of Missouri-Columbia, Columbia, MO 65211 \\ *** State Key Laboratory of Reproductive Biology, Institute of Zoology, Chinese Academy of \\ Sciences, Beijing 100080, P.R. China
}

To understand the regulation of centrosomes and their functional correlation with microtubule organization during pig oocyte activation and fertilization we have studied the organization of the cytoskeleton and the role of the mitogen-activated protein kinase (MAPK). This key regulator of phosphorylation plays critical roles in cell cycle-specific cytoskeletal functions that govern metabolic activities and developmental competence during early and late stages of development. We have analyzed the distribution and functions of the kinase after egg activation, in the dynamically changing centrosome-microtubule complex after fertilization, and in the subsequent symmetric and asymmetric cell divisions during development. Our studies have employed correlative approaches using molecular and imaging techniques including Western immunoblotting, immunofluorescence microscopy, field emission scanning (FESEM) and transmission electron microscopy (TEM).

Centrosomes are microtubule organizing centers that nucleate and define the formation of microtubules. In most animals, except for rodents, dominant centrosome material is contributed by sperm while latent maternal centrosome material is activated during fertilization and unites with the paternal centrosome during nuclear apposition. The proper assembly, functioning and disassembly of microtubules is a prerequisite for the proper completion of oocyte maturation, and centrosomes play an important role in the assembly and formation of the meiotic apparatus, the sperm aster, and the mitotic apparatus during cell division, cell differentiation, and embryo development. The cell cyclespecific regulation of cytoskeletal organization is precisely controlled by specific phosphorylation events. Abnormal centrosome structure and function can be causes for infertility and increases in phosphorylation can be causes for diseases of various nature including cancer [1]. Centrosome and microtubule dynamics in pig oocytes is complex as centrosomes and microtubules carry out a variety of different functions that are central and critical to oocyte activation, fertilization, differentiation, and development. Yet, little is known about the organization of the cytoskeleton and signal transduction that allows the complex structural and metabolic changes.

The mitogen activated protein kinases (MAPKs), are a family of serine/threonine protein kinases that play a key role in microtubule organization. MAP kinase is activated by MAP kinase kinase (MAPKK or MEK) which in turn is activated by Mos protein in the cascade. MAP kinase is present in the spindle and is specifically associated with centrosomes, which makes MAP kinase a candidate for controlling spindle assembly and microtubule configurations.

We have correlated microtubule assembly with the centrosome-specific protein $\gamma$-tubulin and phosphorylated mitogen-activated protein (MAP) kinase in pig oocytes at different maturational stages and during fertilization [2,3,4,5]. The co-localization of $\gamma$-tubulin and phosphorylated MAP kinase with microtubule assembly suggests that MAPK and $\gamma$-tubulin are associated with porcine oocyte centrosomes. We have studied the functions of MAPK by inhibiting its actions with U0126, a 
specific inhibitor of MAP kinase kinase (MEK), the upstream regulator of MAPK actions. The phosphorylation of MAP kinase in oocytes treated with $15 \mu \mathrm{M}$ U0126 was inhibited, while symmetric division was decreased. Our results indicate that 1) MEK plays important roles in microtubule organization; 2) MEK keeps normal meiotic spindle morphology, targets peripheral spindle positioning and regulates asymmetric division.

We have studied the regulation of MAPK phosphorylation and microtubule organization by protein phosphatases during pig oocyte maturation and fertilization in vitro and found that MAPK inhibits spindle organization and suppresses microtubule assembly by sperm centrosomes in pig oocytes and fertilized eggs [5]. We used okadaic acid (OA), a potent inhibitor of protein phosphatases 1 and 2A, to study the regulatory effects of protein phosphatases on mitogen-activated protein (MAP) kinase phosphorylation, morphological nuclear changes, and microtubule assembly during pig oocyte maturation and fertilization in vitro. OA treatment of fertilized eggs resulted in prompt phosphorylation of MAP kinase, disassembly of microtubules around the pronuclear (PN) area, chromatin condensation, and PN membrane breakdown, but inhibited further cleavage. These results suggest that protein phosphatases are major enzymes in the control of MAP kinase activity and that inhibition of protein phosphatases promptly phosphorylates MAP kinase, accelerates premature chromosome condensation, and stimulates interphase exit, but inhibits spindle organization and suppresses microtubule assembly by sperm centrosomes in pig oocytes and fertilized eggs.

Our FESEM and TEM results confirm that centrosomes are contributed by sperm and decompact in the fertilized pig oocyte to form the dynamically changing microtubule configurations that allow apposition of the maternal and paternal nuclei with subsequent mixing of the genome. Studying the regulation of centrosomes and microtubule activity is important as we still do not fully understand the complex interactions on molecular levels that take place during fertilization and we do not fully understand why there are differences during in vitro and in vivo fertilization of pig oocytes. Differences in microtubule organization impacts on mitochondria distribution [3] with consequences for metabolic activity in the fertilized and developing oocyte. The results presented here are steps toward understanding the complex regulation of the cytoskeleton that plays also a critical role during nuclear cloning $[6,7]$.

\section{References}

[1] Schatten, H., Hueser, C.N., and Chakrabarti, A. Microsc. Res. Techn. 49:420-427, 2000.

[2] Sun, Q-Y., Lai, L., Wu, G., Park, K-W., Day, B., Prather, R.S., and Schatten, H. Mol. Reprod. Dev. 60:481-490, 2001.

[3] Sun, Q.-Y., Wu, G.M., Lai, L., Park, K.W., Day, B., Prather, R.S., and Schatten, H. Reproduction 122:155-163, 2001.

[4] Sun, Q.-Y., Lai, L., Prather, R.S., and Schatten, H. Reprod. Fertil. Dev. 12:383-389, 2001.

[5] Sun, Q-Y., Lai, L., Wu, G., Bonk, A., Cabot, R., Park, K-W., Day, B., Prather, R.S., and Schatten, H. Biol. Reprod. 66(3): 580-588, 2002.

[6] Prather, R.S. Science 289:1886-1887, 2000.

[7] Prather RS, Kühholzer B, Lai L, Park K-W. CLONING 2(3): 117-122, 2000.

[8] This research was supported by grants from the University of Missouri-Columbia and Food for the $21^{\text {st }}$ Century Program. 